\title{
The influence of InAs coverage on the performances self-assembled InGaAs quantum rings
}

\author{
Chun-Yuan Huang ${ }^{\mathrm{a}, *}$, Meng-Chyi Wu ${ }^{\mathrm{a}}$, Shih-Yen Lin ${ }^{\mathrm{b}}$, Jong-Horng Dai ${ }^{\mathrm{c}}$, Si-Chen Lee ${ }^{\mathrm{c}}$ \\ ${ }^{a}$ Institute of Electronics Engineering, National Tsing Hua University, Hsinchu, Taiwan \\ ${ }^{\mathrm{b}}$ Opto Electronics and Systems Laboratories, Industrial Technology Research Institute, Hsinchu 310, Taiwan \\ ${ }^{\mathrm{c}}$ Department of Electrical Engineering, National Taiwan University, Taipei, Taiwan
}

Available online 16 December 2006

\begin{abstract}
The influence of InAs coverage on the formation of self-assembled quantum rings (QRs) is investigated in this report. With decreasing InAs coverage from 2.6 to 2.2 monolayers (MLs), the QR density would decrease from $5 \times 10^{9} \mathrm{~cm}^{-2}$ to invisible according to the atomic force microscope (AFM) measurements. The results are attributed to a much smaller 2.2 ML InAs quantum dot (QD) height and therefore much smaller QR structures would be obtained with the almost fully capping GaAs cap layer. Compared with QD sample with the same 2.6 ML InAs coverage, it is found that the QD density is about one order of magnitude larger than that of QRs. The phenomenon is attributed to the QD size non-uniformity such that instead of partial capping, the 2-nm-GaAs cap layer has fully covered the smaller QDs. Also observed is the blue shift of photoluminescence (PL) peak wavelengths of the QR structures with decreasing InAs coverage, which is attributed to the increase of Ga composition and the decrease of QR height during the intermixing of GaAs capping layer and the InAs QDs.

(C) 2007 Elsevier B.V. All rights reserved.
\end{abstract}

Keywords: A1. Atomic force microscopy; A1. Nanostructures; A3. Molecular beam epitaxy

\section{Introduction}

Due to the unique ring-like structures, self-assembled InGaAs quantum rings (QRs) prepared by molecular beam epitaxy (MBE) have attracted much attention in recent years [1,2]. Because the QR structures are formed via postgrowth annealing of partially GaAs-capped InAs quantum dots (QDs), similar growth procedures and quantum size effect are observed for both structures. However, different from QD structures, QR structures are of not simply connected geometry. This geometry makes QRs particularly suitable for studies in magnetic fields [3-5]. According to the previous reports, two mechanisms have been proposed to explain the origin of the ring formation: (a) the diffusion-driven transformation from QD to QR structures and (b) the dewetting process at the post-growth annealing procedure. Neither of these two mechanisms can perfectly explain all the phenomena observed during the

\footnotetext{
${ }^{*}$ Corresponding author. Tel.: + 8863571513134028.

E-mail address: d919004@alumni.nthu.edu.tw (C.-Y. Huang).
}

ring formation. Basically, the former comes from the kinetic effect and is suitable to interpret the QR formation in InAs/GaAs material system. Experimentally, the formation of InGaAs QR structure is based on QD structure with an additional thin GaAs capping layer and following postgrowth annealing procedure $[2,6]$. The demonstration by Garcia et al. [2], showed a large anisotropic redistribution of the InAs taking place during the initial GaAs covering, which results in the gradual out diffusion of the uncapped InAs material. As for the special characteristics of the QR structures, it is reported that the QR structures are of large and negative excitonic permanent dipole moments [7], high oscillator strength of the band-to-band ground state transition [8], polarizability and magnetic properties [4,5]. The characteristics have made the structures advantageous for novel device developments.

Due to the significant influence of the preformed InAs QDs on the InGaAs QRs, the influence of InAs coverage on the formation of self-assembled QRs is investigated. In this report, both the surface morphologies and optical characteristics of QR and QD samples are analyzed. The 
surface morphological properties were characterized by ex situ tapping mode atomic force microscopy (AFM) while the optical characteristics were evaluated by photoluminescence (PL) measurements. The PL measurements were performed using a diode pumped green laser which emitted at $532 \mathrm{~nm}$. The luminescence is dispersed by a $0.5 \mathrm{~m}$ monochromator, and detected by an InGaAs PIN detector. The samples are cooled down to $15 \mathrm{~K}$ in a liquid helium cryostat.

\section{Experimental results}

The QR samples discussed in this report are prepared by VG-80 H solid-source MBE system on (100)-oriented GaAs substrates. As shown in Table 1, all the samples are composed of a pair of InGaAs/GaAs QR structures with one embedded in the GaAs matrix for PL measurements and the other on the GaAs surface for AFM measurements. Samples with decreasing InAs coverage from 2.6 to 2.2 monolayer (ML) are referred as samples A-E, respectively. The QR structures are formed via $30 \mathrm{~s}$ at $480{ }^{\circ} \mathrm{C}$ post-growth annealing of $2 \mathrm{~nm}$ GaAs partial capping layers over the preformed InAs QDs prepared under Stranski-Krastanov growth mode. The growth rates for InAs and GaAs are $0.26 \mathrm{ML} / \mathrm{s}$ and $1 \mu \mathrm{m} / \mathrm{h}$, respectively.

The $1 \times 1 \mu \mathrm{m}^{2} \mathrm{AFM}$ results of QR samples are shown in Figs. 1(a)-(e). Also shown in Fig. 1(f) is the $0.5 \times 0.5 \mu \mathrm{m}^{2}$ AFM image of the QD reference sample with 2.6 ML InAs coverage. As shown in the figure, the QR density would decrease from $5 \times 10^{9} \mathrm{~cm}^{-2}$ to invisible from sample A to E with decreasing InAs coverage. Here, the "invisible" means we cannot observe any features by AFM within a certain area, however, it does not mean the density of QRs is zero. Also observed are the incomplete ring shapes. Most of the QRs merely reveal the central crater-like depressions. Consequently, the size and height of QRs are unable to be estimated due to the floating bases. Since the QRs are transformed from QDs, the QD and QR samples with the same InAs coverage such as sample A and the reference QD sample are compared. As shown in the AFM image Fig. 1(f) of the reference sample, average size of the lensshaped QDs is of $20 \mathrm{~nm}$ in diameter and $6 \mathrm{~nm}$ in height. The QD density is $6 \times 10^{10} \mathrm{~cm}^{-2}$, which is one order

Table 1

The QR sample structures with different InAs coverages

\begin{tabular}{|c|c|c|c|c|c|}
\hline & A & B & $\mathrm{C}$ & $\mathrm{D}$ & $\mathrm{E}$ \\
\hline InAs layer at $480^{\circ} \mathrm{C}$ & \multicolumn{5}{|c|}{$2 \mathrm{~nm} \mathrm{GaAs}$ at $480^{\circ} \mathrm{C}$} \\
\hline Thickness (ML) & 2.6 & 2.5 & 2.4 & 2.3 & 2.2 \\
\hline & \multicolumn{5}{|c|}{$\begin{array}{l}60 \mathrm{~nm} \text { GaAs at } 480^{\circ} \mathrm{C} \\
5 \mathrm{~nm} \mathrm{GaAs} \text { at } 430^{\circ} \mathrm{C}\end{array}$} \\
\hline InAs layer at $480^{\circ} \mathrm{C}$ & \multicolumn{5}{|c|}{$2 \mathrm{~nm} \mathrm{GaAs}$ at $480^{\circ} \mathrm{C}$} \\
\hline Thickness (ML) & 2.6 & 2.5 & 2.4 & 2.3 & 2.2 \\
\hline & \multicolumn{5}{|c|}{$\begin{array}{r}0.2 \mu \mathrm{m} \mathrm{GaAs} \text { buffer layer at } 600^{\circ} \mathrm{C} \\
\text { GaAs (100) substrate }\end{array}$} \\
\hline
\end{tabular}
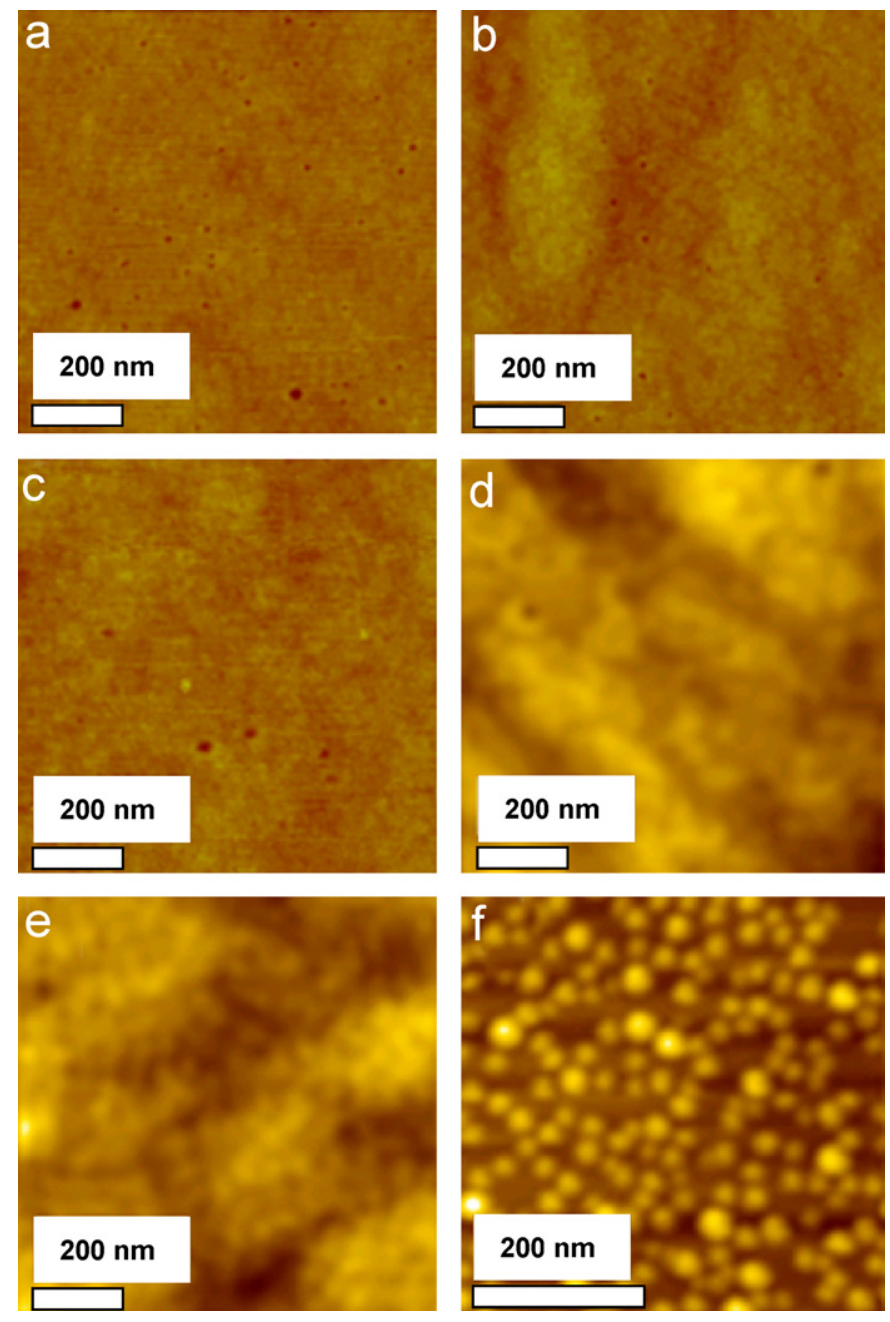

Fig. 1. (a)-(e) The $1 \times 1 \mu \mathrm{m}^{2}$ AFM images of QR samples with different InAs coverages $2.6-2.2 \mathrm{ML}$ and (f) The $0.5 \times 0.5 \mu \mathrm{m}^{2}$ AFM image of the QD reference sample with $2.6 \mathrm{ML}$ InAs coverage.

of magnitude larger than the QR density of sample A. According to previous report, the well-defined InGaAs QRs can be observed only when the density of uncapped QDs is less than $5 \times 10^{9} \mathrm{~cm}^{-2}$ [2]. The influence of QD density is illustrated in Fig. 2. Generally speaking, a higher QD density would indicate a shorter distance between different dots. In Fig. 2(a), a moderate density of QDs can make the QDs perfectly transform into QRs with wellresolved ring shape due to the sufficient distance between QDs. However, too high a density could make a QR coalesce with nearby ones. As a result, only the central crater-like depression of QRs can be identified on the AFM images. The phenomenon is responsible for the indistinct shape of the QR structures. It should be noted the vertical black-to-white contrast in Figs. 1(a)-(c) is $4 \mathrm{~nm}$, in Figs. 1(d) and (e) is $2 \mathrm{~nm}$, and in Fig. 1(f) is $8 \mathrm{~nm}$, respectively.

To further explain the obvious reduction of QR density compared with the QD reference sample, the line profiles of dots and rings are shown in Fig. 3. As shown in the figure, the profile of the reference QD sample exhibits the partial 
a

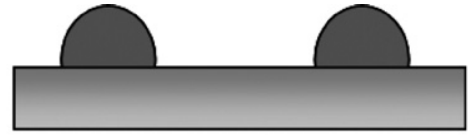

b

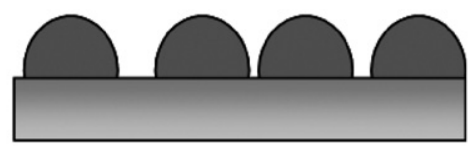

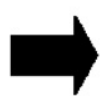
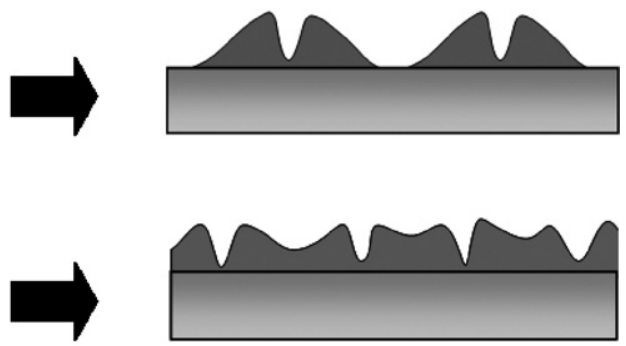

Fig. 2. The illustration of the influences of QD distance on the QR formation: (a) QDs with lower density would result in well-defined QRs, and (b) QDs with higher density would result in QR coalescence during post-growth annealing procedure.

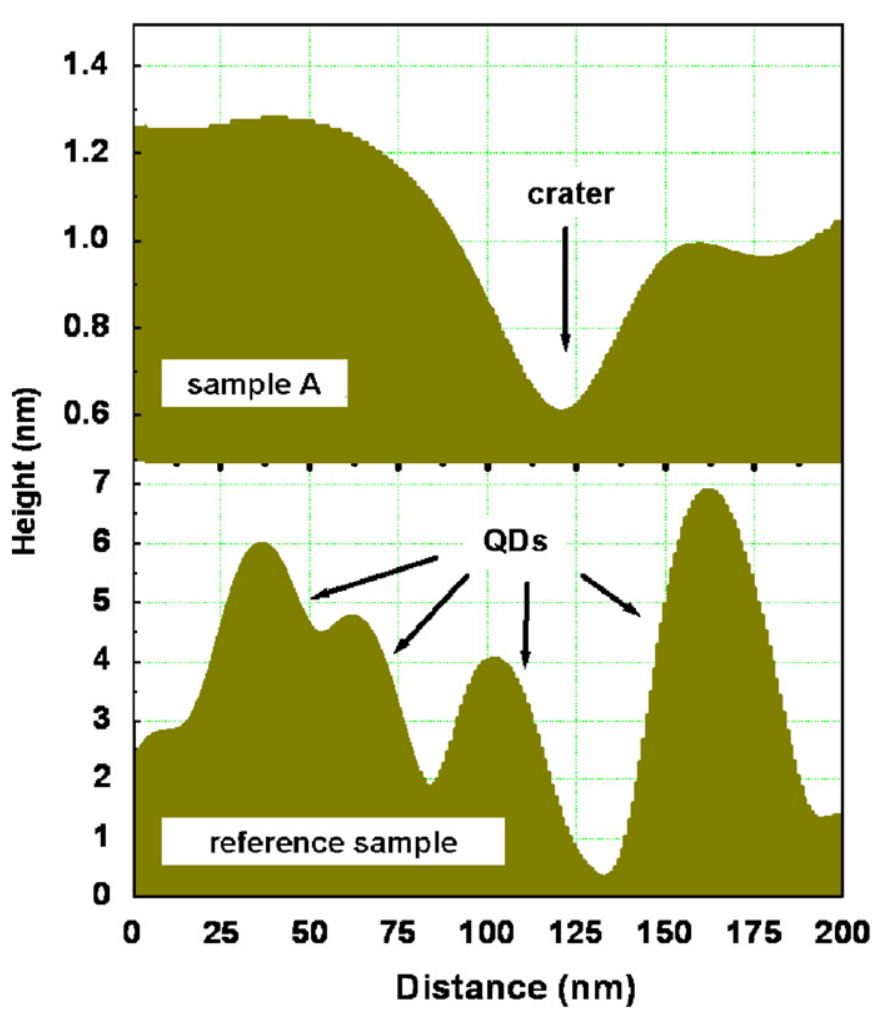

Fig. 3. The scanned profiles of sample A and the reference sample from AFM images.

overlap of adjacent QDs, which would relatively reduce the vertical height of dots. Under such a situation, the $2 \mathrm{~nm}$ GaAs layer would, instead of partially capping, almost fully cap the smaller QDs. Therefore, these overlapped QDs as well as the small dots originated from size fluctuation are responsible for the observed QR density reduction. Also shown in Fig. 3 is the profile of sample A. As shown in the figure, the only part of a $\mathrm{QR}$ structure that we can recognize is the central crater-like depression. The measured depth of the crater-like depression for sample A is typically less than $1 \mathrm{~nm}$, which is underestimated due to the floating bases and the finite size of the AFM tip. As shown in previous reports, the depth of the crater-like depression would decrease with increasing the thickness of GaAs capping layer, and finally, this feature disappeared. The results suggest that larger QDs are a necessary precondition for the formation of well-defined rings
[9-11]. From this conclusion, it is reasonable to assume that in samples D and E, the $2 \mathrm{~nm}$ GaAs capping layer has almost completely covered all the dots and flattens the surface. Even if a small proportion of QDs is partially covered, the formed QRs after annealing are too small to be well evaluated by AFM.

The $15 \mathrm{~K}$ PL spectrums of all the samples are shown in Fig. 4. While comparing the spectra of sample A and reference sample, only the excitonic ground state transition from the ensemble QRs and QDs can be observed. The identical integrated PL intensities indicate the similar threedimensional confinements as well as carrier capturing efficiency in both of them. Nonetheless, the ground state transition from QRs is blueshifted $\sim 28 \mathrm{meV}$ with respect to that from QDs with the same InAs coverage, which is much smaller than the previously reported [2,9]. Moreover, the full-width at half-maximum (FWHM) of the later is remarkably larger than that of the former. It should be attributed to the improvement of QR size distribution with respect to the QDs during the GaAs capping and annealing process. Supposedly, the unapparent PL tail at low energy side of ground state transition for sample A can be attributed to the luminescence of the residue shapemodified QDs.

Meanwhile, the photon energy of the ground state transition of samples $\mathrm{A}-\mathrm{C}$ is around $1.23 \mathrm{eV}$ and the FWHM of this transition are 70,65 , and $60 \mathrm{meV}$, respectively. Again, the decrease of FWHM is attributed the improvement of QR size uniformity since the inhomogeneous broadening of PL is inherited from the size distribution of QDs. Also observed is the increase of integrated PL intensity with decreasing InAs coverage for samples $\mathrm{A}-\mathrm{C}$, which is attributed to the enhancement of carrier confinement resulted from height reduction. The QR density seems to have a minor contribution to PL intensity since that sample A has the highest density.

In contrast with the spectra of samples $\mathrm{A}-\mathrm{C}$, the blue shifts of ground state luminescence are observed for samples $\mathrm{D}$ and $\mathrm{E}$. This phenomenon is attributed to the relative increase of $\mathrm{Ga}$ composition during the In-Ga intermixing process resulted from the smaller InAs QDs with lower InAs coverage. Accompanying with the shift of peak wavelengths, the reduction of integrated PL intensity can be directly related to the much lower QR density as shown in the AFM images. Also observed in the PL spectra 


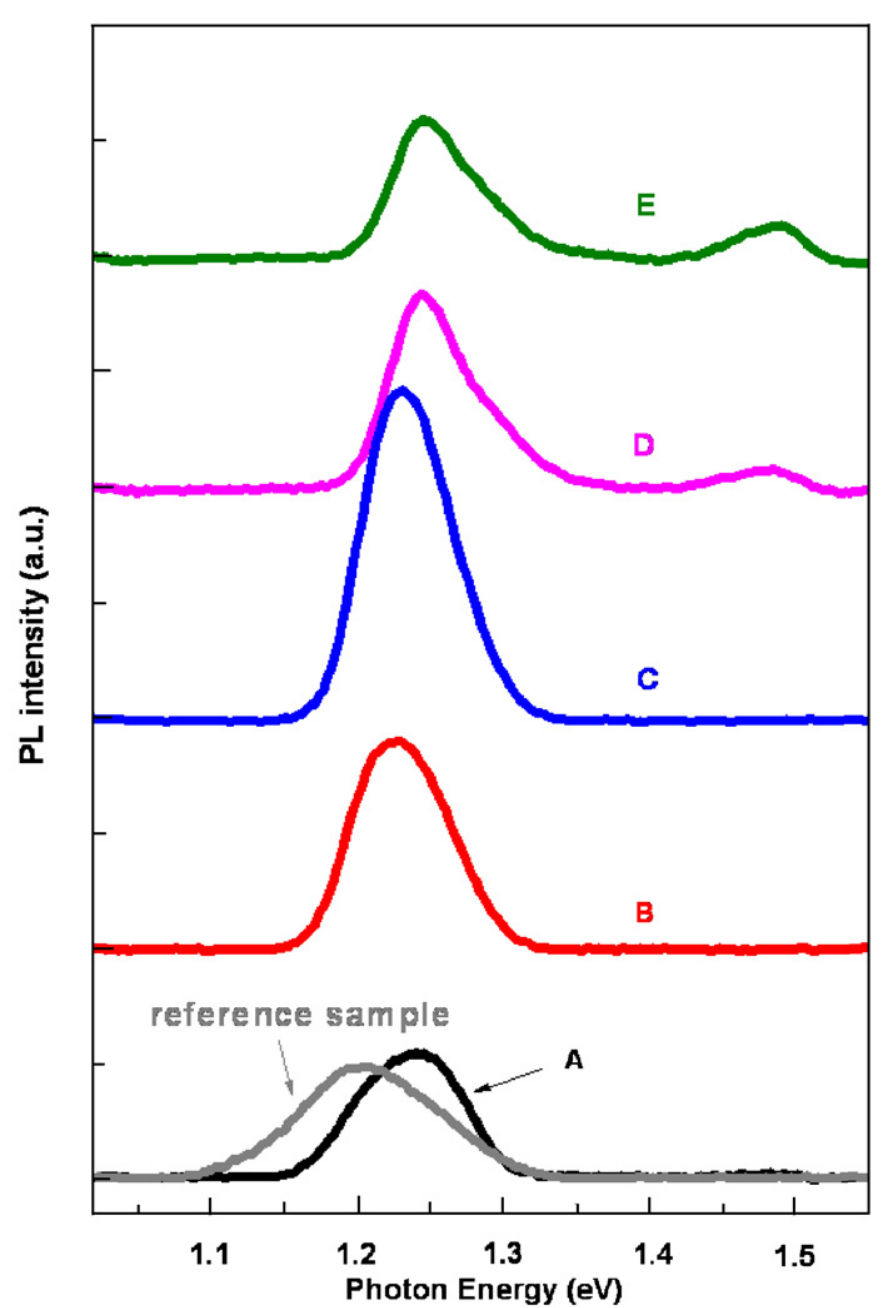

Fig. 4. The $15 \mathrm{~K}$ PL spectra for samples A-E, as well as the reference sample. Parts of spectra are vertically offset for clarity.

of samples $\mathrm{D}$ and $\mathrm{E}$ is the appearance of wetting layer signal at $1.49 \mathrm{eV}$. The appearance of wetting signal is consistent with the observation of $\mathrm{QR}$ density reduction [12]. Besides, the spectra of sample D and E have a broad high-energy tail. These tail states behave-like bound quantum-well exciton transition since they are gradually quenched at higher temperature in temperature-varying PL measurement. Basically, the smaller QDs have merged with the GaAs capping layer and become InGaAs quantum-well structure instead of QR formation.

The temperature-varying PL measurements were performed from 15 to $150 \mathrm{~K}$. The integrated PL intensities as a function of temperature are shown in Fig. 5. As shown in the figure, all samples exhibit anomalous intensity increase with increasing temperature. The phenomenon suggests that interactions between excited electrons and phonons are not dominant at temperatures below $100 \mathrm{~K}$. The similar phenomenon has also been reported in the optically characteristics of ensemble QDs [13-15]. It is reasonable to conclude that it is associated with the presence of wetting layer in the QR samples.

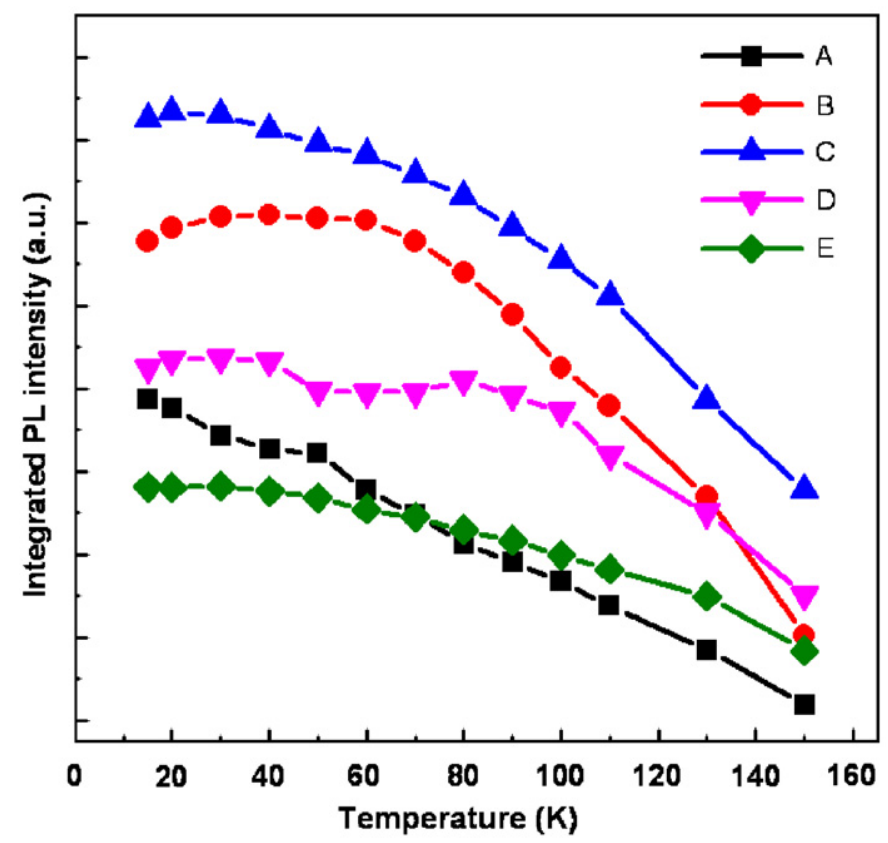

Fig. 5. The integrated PL intensities as functions of temperature for samples A-E.

\section{Conclusions}

In conclusion, the influence of InAs coverage on the formation of self-assembled quantum rings is investigated in this report. With decreasing InAs coverage from 2.6 to 2.2 ML, the QR density would decrease from $5 \times 10^{9} \mathrm{~cm}^{-2}$ to invisible according to the AFM measurements. The results are attributed to a much smaller 2.2 ML InAs QD height and therefore much smaller QR structures would be obtained with the almost fully capping GaAs cap layer. Compared with QD sample with the same 2.6 ML InAs coverage, it is found that the QD density is about one order of magnitude larger than that of QRs. The phenomenon is attributed to the QD size non-uniformity such that instead of partial capping, the $2 \mathrm{~nm}$ GaAs cap layer has fully covered the smaller QDs. Also observed is the blue shift of PL peak wavelengths of the QR structures with decreasing InAs coverage, which is attributed to the increase of Ga composition and the decrease of $\mathrm{QR}$ height during the intermixing of GaAs capping layer and the InAs QDs. It is concluded that to obtain the well-resolved QRs, the reduction of QD density and the increase of QD size before the thin GaAs cap layer deposition are necessary. Also, a uniform QD distribution is helpful to shrink the density difference between QDs and QRs with the same InAs coverage.

\section{References}

[1] L. Landin, M.S. Miller, M.-E. Pistol, C.E. Pryor, L. Samuelson, Science 280 (1998) 262.

[2] J.M. Garcia, G. Medeiros-Ribeiro, K. Schmidt, T. Ngo, J.L. Feng, A. Lorke, J. Kotthaus, P.M. Petroff, Appl. Phys. Lett. 71 (1997) 2014. 
[3] J. Planelles, W. Jaskolski, J.I. Aliaga, Phys. Rev. B 65 (2002) 033306.

[4] Z. Barticevic, M. Pacheco, A. Latge, Phys. Rev. B 62 (2000) 6963.

[5] O. Voskoboynikov, Y. Li, H.M. Lu, C.F. Shih, C.P. Lee, Phys. Rev. B 66 (2002) 155306.

[6] A. Lorke, J.M. Garcia, R. Blossey, R.J. Luyken, P.M. Petroff, Adv. Solid State Phys. 43 (2003) 125.

[7] R.J. Warburton, C. Schulhauser, D. Haft, C. Schaflein, K. Karrai, J.M. Garcia, W. Schoenfeld, P.M. Petroff, Phys. Rev. B 65 (2002) 113303.

[8] H. Pettersson, R.J. Warburton, A. Lorke, K. Karrai, J.P. Kotthaus, J.M. Garcia, P.M. Petroff, Physica E 6 (2000) 510.

[9] D. Granados, J.M. Garcia, Appl. Phys. Lett. 82 (2003) 2401.
[10] R. Songmuang, S. Kiravittaya, O.G. Schmidt, J. Crystal Growth 249 (2003) 416.

[11] T. Raz, D. Ritter, B. Bahir, Appl. Phys. Lett. 82 (2003) 1707.

[12] C.-Y. Huang, T.-M. Ou, S.-T. Chou, C.-S. Tsai, M.-C. Wu, S.-Y. Lin, J.-Y. Chi, J. Vacuum Sci. Technol. B 23 (2005) 1909.

[13] S. Sanguinetti, M. Henini, M.G. Alessi, M. Capizzi, P. Frigeri, S. Franchi, Phys. Rev. B 60 (1999) 8276.

[14] S. Sanguinetti, T. Mano, M. Oshima, T. Tateno, M. Wakaki, N. Koguchi, Appl. Phys. Lett. 81 (2002) 3067.

[15] W. Yang, R.R. Lowe-Webb, H. Lee, P.C. Sercel, Phys. Rev. B 56 (1997) 13314. 